\title{
On the effect of the initial magnetic polarity and of the background wind on the evolution of CME shocks
}

\author{
E. Chané, C. Jacobs, B. Van der Holst, S. Poedts, and D. Kimpe
}

\author{
Centre for Plasma Astrophysics, Katholieke Universiteit Leuven, Celestijnenlaan 200B, 3001 Leuven, Belgium \\ e-mail: Emmanuel. Chane@wis . kuleuven .ac. be
}

Received 14 September 2004 / Accepted 3 November 2004

\begin{abstract}
The shocks and magnetic clouds caused by Coronal Mass Ejections (CMEs) in the solar corona and interplanetary (IP) space play an important role in the study of space weather. In the present paper, numerical simulations of some simple CME models were performed by means of a finite volume, explicit solver to advance the equations of ideal magnetohydrodynamics. The aim is to quantify here both the effect of the background wind model and of the initial polarity on the evolution of the IP CMEs and the corresponding shocks.

To simulate the CMEs, a high density-pressure plasma blob is superposed on different steady state solar wind models. The evolution of an initially non-magnetized plasma blob is compared with that of two magnetized ones (with both normal and inverse polarity) and the differences are analysed and quantified. Depending on the launch angle of the CME and the polarity of the initial flux rope, the velocity of the shock front and magnetic cloud is decreased or increased. Also the spread angle of the $\mathrm{CME}$ and the evolution path of the CME in the background solar wind is substantially different for the different CME models and the different wind models. A quantitative comparison of these simulations shows that these effects can be quite substantial and can clearly affect the geo-effectiveness and the arrival time of the events.
\end{abstract}

Key words. Sun: solar wind - Sun: coronal mass ejections (CMEs) - magnetohydrodynamics (MHD)

\section{Introduction}

It is well-known that Coronal Mass Ejections (CMEs) play an important role in space weather. Therefore, a careful study of the origin, the structure, and the propagation characteristics of these violent phenomena is essential to get a deeper insight in space weather physics. This insight is crucial for better, more accurate space weather predictions.

CME shock evolution studies very often apply simple generation models for the CMEs. Jacobs et al. (2004, hereafter Paper I) superposed a CME on their 2.5D MHD wind solution. They used the simple "density(+pressure)-driven" model and simulated CMEs both in the equatorial streamer belt and at larger latitudes. Groth et al. (2000) also simulated "densitydriven" CMEs superposed on a 3D ideal MHD wind solution and studied their interaction with the magnetosphereionosphere system (see also Gombosi et al. 2000). Roussev et al. (2003) considered the Titov \& Démoulin flux rope model as initial condition although the evolution of this CME is only simulated in the lower corona. Manchester et al. (2004a) used the same wind solution but superposed an analytical, 3D, "Gibson \& Low (1998) family" self-similar CME in the streamer belt and followed its evolution up to $32 R_{\odot}$. It is found that the flux rope expands rapidly and drives a strong shock ahead of it. As the CME evolves in interplanetary (IP) space, the flux rope is deformed and the MHD shock front propagates at the same speed as the flux rope in the center but far ahead of it at the sides of the rope. In another paper, Manchester et al. (2004b) focussed on a space weather event simulating a CME from its formation at the Sun through its evolution in IP space up to $336 R_{\odot}$. Other simple models generate a pressure pulse with or without an additional velocity change (i.e. a kind of "nozzle" boundary condition), see e.g. Wang et al. (1995); Odstrcil \& Pizzo (1999). Keppens \& Goedbloed (1999) on the other hand impose an extra mass flow to generate the CMEs.

Odstrcil \& Pizzo (1999) also first used simple CME models (an over-pressured spherical plasmoid) and followed the CME for a long time (up to $5 \mathrm{AU}$ ). They showed that the CME interacts strongly with the co-rotating streamer belt and that this interaction can be very complex. Later, Odstrcil \& Pizzo (2002) used a more realistic analytical flux-rope model for the CME and studied the compression of the flux rope and the evolution of the dimpled leading shock front (see also Odstrcil et al. 2003). Odstrcil et al. (2002) demonstrated the merging of coronal and heliospheric 2D MHD models developed at SAIC San Diego and CU/CIRES-NOAA/SEC Boulder. These models are quite different as regards the included physics and the used numerical techniques and grids over their respective spatial and temporal domains. Odstrcil et al. (2003) also presented results of ongoing coupled 3D MHD simulations of a fastmoving magnetic flux rope that stretches from the Sun through the model interface boundary into the heliosphere. 
However, a serious problem arises when one tries to compare the results of two or more of these studies because different background wind models are used, as well as different CME initiation models. Moreover, different authors often use completely different numerical techniques with different numerical dissipation and different shock capturing properties. In the present paper, we want to quantify, in an objective way, the influence of both the background wind model and the magnetic polarity of the flux-rope CME model. Three frequently used wind models will be reconstructed and three simple CME models will be superposed on these winds. All the simulations discussed in the present paper are obtained with the same numerical code, the same grid resolution (numerical dissipation), and the same boundary conditions. All the solutions presented are 2.5D (axisymmetric) and are obtained with the total variation diminishing Lax-Friedrichs solver in Versatile Advection Code (VAC, see Tóth 1996). In Paper I, we focused on the effect of the background wind on the evolution properties of fast CMEs and the shocks they generate. The same simple CME model was superposed on the three wind models. In the present paper, we consider the same wind models but concentrate on the magnetic properties of the CME and their effect on the evolution of the CMEs in the IP space. Our wind model 1 is a polytropic wind with $\gamma=1.05$ (based on Keppens \& Goedbloed 1999). Wind model 2 is an MHD wind model with and additional energy source term (based on Groth et al. 2000) in order to better model the observed wind speeds. Wind model 3 is a polytropic wind model with an Alfvén wave pressure term (see Paper I for more details about the wind models).

In the present paper, the effect of the magnetic polarity of the CME flux rope on the CME evolution is investigated. We simulate three CME models superposed on the same background wind (model 1). The different CME models are presented in Sect. 2. In Sect. 3, we focus on the effects of the initial magnetic polarity. The similarities between our results and the theoretical model of Low \& Zhang (2002) are shown and discussed. The characteristic shapes of the different CMEs are pointed out. It appears that the initial polarity influence the propagation speed, the evolution path and the spread angle. In Sect. 4, the effects of the background wind on the CME evolution are shown.

\section{Initiation}

In order to study the propagation of MHD shocks in the interplanetary space, a very simple CME model is used for generating the shocks. Although the initiation model is rather simplistic, different background winds and initial magnetic polarities lead to substantially different results.

In the simulations discussed in the present paper, a blob of high density and high pressure is created and launched with high velocity in outward radial direction, superposed on the steady solar wind models mentioned before. The radius of the initial plasma blob is $0.29 R_{\odot}$ and its center is located at $1.5 R_{\odot}$. In the center of the initial perturbation, the velocity is about $1000 \mathrm{~km} \mathrm{~s}^{-1}$ and the density is 5 times higher than the density on the surface of the sun (see Paper I).
In Paper I, the initial plasma blob was unmagnetized. In the present paper, we consider a magnetized plasma blob and quantify the effect of the initial magnetic field polarity on the evolution of the IP CME. The structure of the poloidal components $B_{R}$ and $B_{Z}$ of the initial magnetic field is prescribed by means of a poloidal magnetic flux $\psi$ :

$$
B_{R}=-\frac{1}{R} \frac{\partial \psi}{\partial Z}, \quad B_{Z}=\frac{1}{R} \frac{\partial \psi}{\partial R} .
$$

The magnetic flux profile is chosen in such a way that the added CME magnetic field is smoothly connecting to the background wind:

$\psi=\psi_{1}\left(d-\frac{d_{\mathrm{cme}}}{2 \pi} \sin \frac{2 \pi d}{d_{\mathrm{cme}}}\right)$,

with $d_{\text {cme }}$ the radius of the perturbation (here $d_{\text {cme }}=0.29 R_{\odot}$ ), and $d$ the distance to the center of this perturbation.

Depending on the value of the constant $\psi_{1}$, three different CMEs can be created:

- when $\psi_{1}=0$, there is no extra magnetic field (this case is treated extensively in Paper I);

- when $\psi_{1}<0$, the magnetic field of the CME has initially the same polarity as the magnetic field of the background solar wind. This situation is called an inverse CME (according to the nomenclature of Low \& Zhang 2002);

- when $\psi_{1}>0$, the magnetic field of the CME has initially the opposite polarity compared to the background magnetic field. This case is called a normal CME (according to the nomenclature of Low \& Zhang 2002).

Figure 1 shows these three different magnetic perturbations superposed on the wind model 1.

In this paper a comparison between these three cases will be carried out. The three CME models will be superposed on the three background wind models and the main differences in evolution properties will be pointed out and quantified. In all numerical simulations discussed here, the CMEs have exactly the same initial velocity, the same initial density and the same initial pressure. Also the numerical resolution and the numerical technique is exactly the same in all simulations. The only difference will be the combination of the initial magnetic field configuration and the background solar wind model. The maximal magnetic field strength that we consider in the initial plasma blob is $\sim 3.7$ Gauss for both the normal and the inverse case. Even for such a small perturbation, it will be shown that the initial magnetic polarity plays an important role in the evolution of the CME in IP space.

\section{Effect of the magnetic polarity on the CME evolution}

We first analyze and quantify where possible the effect of the initial flux rope magnetic field and, in particular, its polarity (with respect to the background solar wind magnetic field) on the evolutionary features of the CMEs in IP space. In this section, we only consider the polytropic solar wind (model 1) as background wind model. 

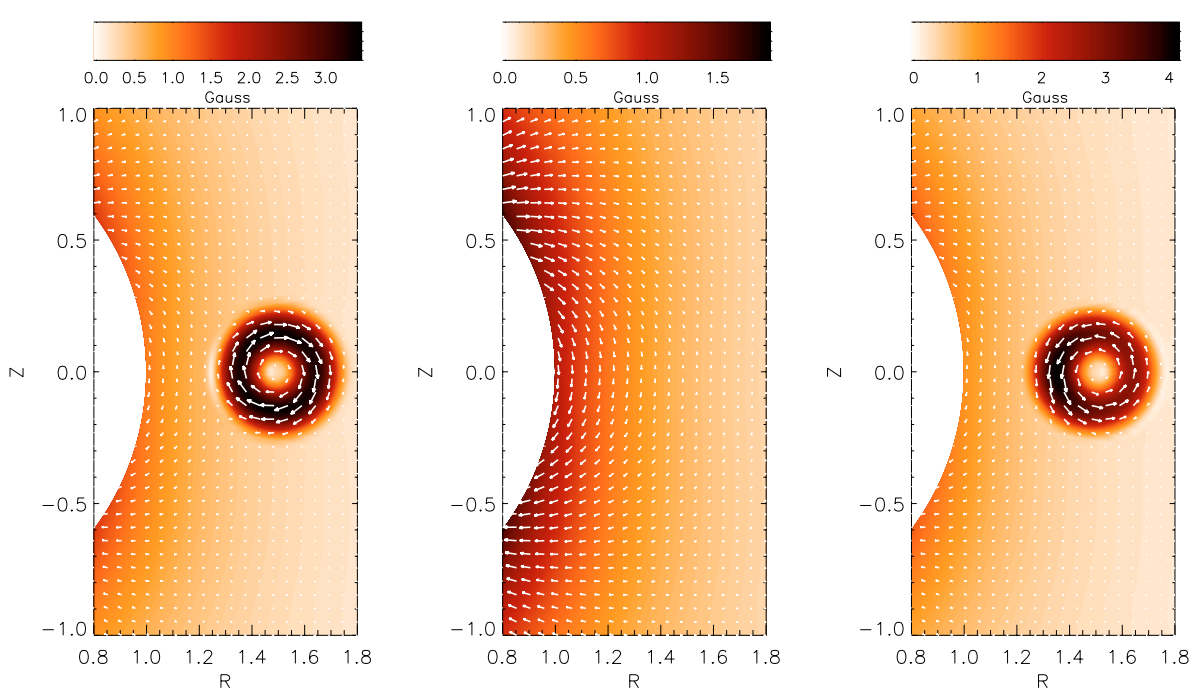

Fig. 1. Direction and strength of the initial magnetic field; left: inverse polarity $\left(\psi_{1}<\right.$ $0)$; middle: unmagnetized $\left(\psi_{1}=0\right)$; right: normal polarity $\left(\psi_{1}>0\right)$. Shown are the magnetic field vectors and a contour plot of the magnetic field strength (close-up of the initial perturbation).
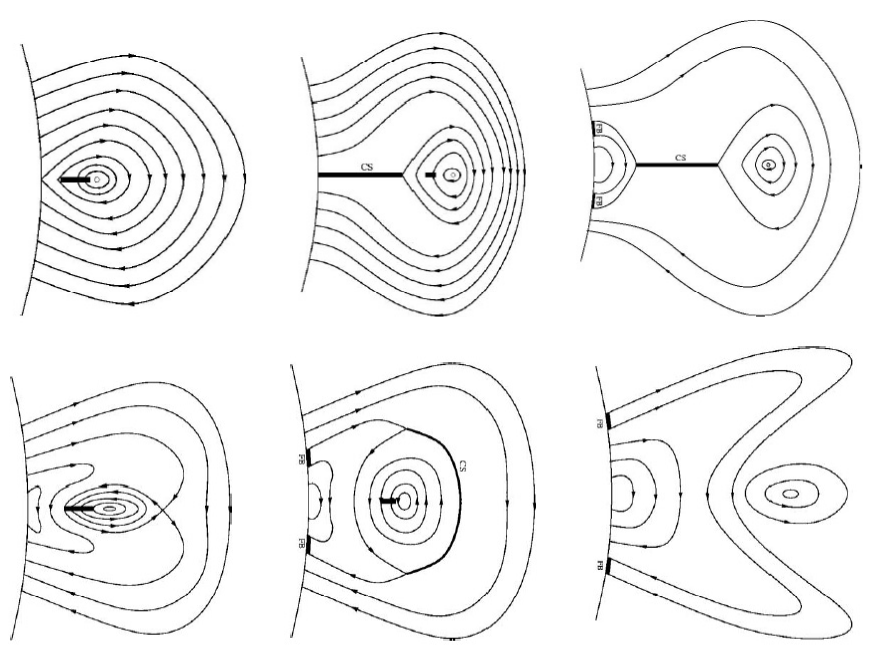

Fig. 2. Evolution in time of the poloidal projections of the magnetic field lines of the two different CME models (Zhang \& Golub 2003); above: the inverse CME configuration; below: the normal CME configuration.

\subsection{The magnetic field configuration}

Low \& Zhang (2002) have established a qualitative theoretical CME model that makes a distinction between two kinds of CMEs: the normal and the inverse CME, depending on the polarity of the magnetic field in the corresponding quiescent prominences compared to that of the background solar wind. For the normal CME, the magnetic field in the flux rope circulates in the opposite sense relative to the surrounding coronal magnetic field. For the inverse CME, the magnetic field in the initial massive prominence sheet in the flux rope circulates in the same sense as the magnetic field in the dense coronal mass outside the flux rope.

Figure 2 shows the qualitative evolution in time of this theoretical model for these two cases. In the case of the inverse configuration, the lift-off of the flux rope is initiated by the loss of mass to the prominence and creates a current sheet behind the flux rope. After the flux rope expulsion, magnetic reconnection produces closed bipolar fields anchored to the atmospheric base and gives rise to a pair of foot point brightenings due to the newly reconnected flux, corresponding to the two-ribbon flare. For the normal configuration, the lift-off of the flux rope owing to mass-loss to the prominence forms a current sheet ahead of the flux rope. After the flux rope expulsion, the new sling-shot field topology (created by the magnetic reconnection that removed the fields ahead of the flux rope) again produces a pair of foot point brightenings belonging to the newly reconnected flux and corresponding to the two-ribbon flare.

Somewhat surprisingly, the simple CME initiation method used in our numerical simulations easily reproduces the two dynamical types of solar CMEs described by Low \& Zhang (2002). For comparison Fig. 3 shows our results after 4h12min of propagation: the magnetic field configuration is in agreement with the qualitative theoretical model of Low \& Zhang (2002), including the formation of the current sheet behind the flux rope in the inverse configuration. The evolution of the initially unmagnetized CME is a kind of "average" between inverse an normal cases, with the magnetic field topology looking more like that of the inverse case but with a density distribution looking closer to that in the normal CME. Clearly, during the propagation of the initially unmagnetized perturbation, the magnetic field of the sun is also disturbed and the CME carries away a part of this magnetic field.

Note that we considered the ideal MHD equations. Nevertheless, due to the discretization, numerical dissipation occurs in our simulations. As a result, magnetic field lines can reconnect as in the real corona. Due to the limited numerical resolution, however, the numerical dissipation is much larger that the real dissipation in the highly conductive solar corona. Therefore, the magnetic reconnection events and the formation of current sheets observed in our results are more qualitative than quantitative and we do not pay too much attention to them in the analysis of the results.

\subsection{The characteristic shape}

As it is clear from the previous subsection, the flux rope magnetic field affects the evolution of the characteristic shape of the CMEs, i.e. the distribution of the plasma density in the 

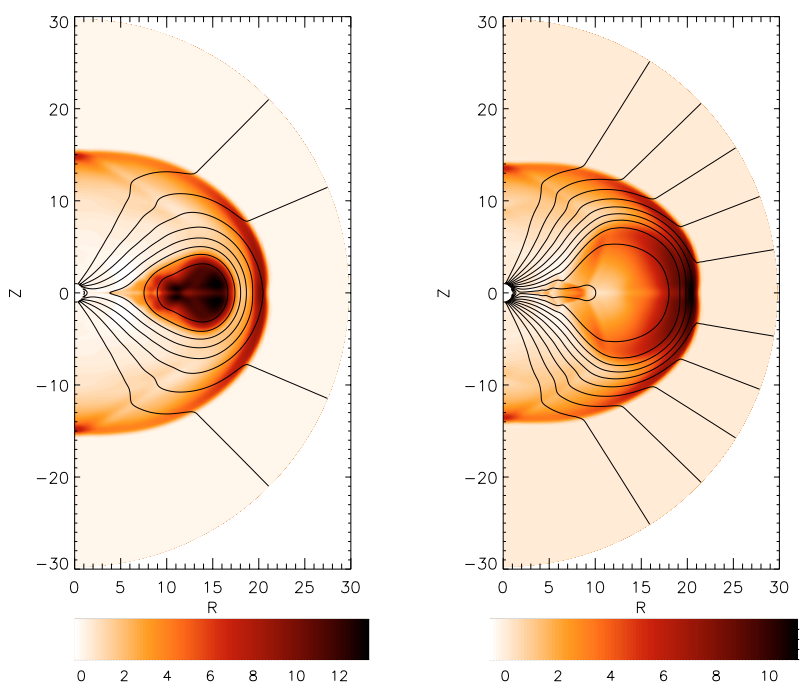

Fig. 3. Plots of the relative density profile after $4 \mathrm{~h} 12 \mathrm{~min}$ for a CME launched in the equatorial plane $\left(\theta_{\mathrm{cme}}=0^{\circ}\right)$; left: magnetized inverse; middle: unmagnetized; right: magnetized normal. The black lines represent the magnetic field.
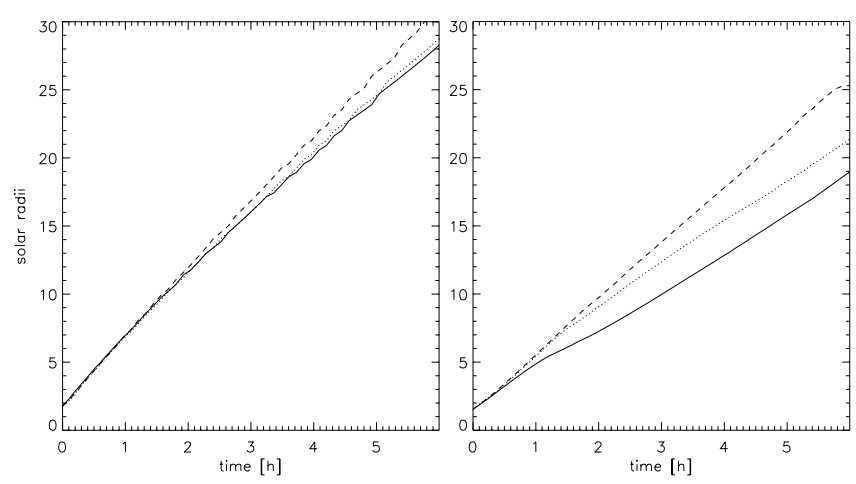

Fig. 4. Height-time plot of the shock front position (left panel) and of the center of relative mass position (right panel) on the equatorial plane; dotted line: unmagnetized CME; dashed line: normal CME; solide line: inverse CME.

CME. In this subsection we will study this effect and we try to point out the characteristic plasma shapes resulting from the three models. As it is clear from Fig. 3 the density distribution (which is initially exactly the same) evolves totally differently for the three cases. For the perturbation with the inverse magnetic field polarity the bulk of the CME plasma is trapped in the central flux rope and more or less maintains its initially circular shape. Also, in this case the plasma blob is clearly detached from the leading shock front. On the other hand, for the perturbation with the normal magnetic polarity and for the initially unmagnetized perturbation, the CME plasma does not maintain its circular shape. Instead the perturbation is flattened as it evolves towards a "pancake" shape immediately following the leading shock front.

Let us now try to find an explanation for the fact that these three similar models evolve to such substantially different shapes. Clearly, the reason for this dissemblance must be due to the differences in the initial magnetic pressure since the initial magnetic field is the only unlikeness in the three models. The magnetic force is directed from high magnetic field strength regions toward the regions with a lower magnetic field strength. Keeping this in mind, let us have a closer look at Fig. 1. In the left panel, i.e. for the inverse CME launched on the equator, at the start of the simulation the bulk part of the CME plasma is located in the middle of a kind of magnetic jail, i.e. it is completely surrounded by high magnetic field. In response to the initial velocity the CME propagates outward from the Sun but it is impossible for the plasma to escape from this magnetic jail. Moreover, the magnetic pressure progressively separates the leading shock front from the center of mass.

For the normal CME, on the other hand, one can see in Fig. 1 that the initial magnetic field strength is larger at the side of the plasma blob near the Sun than at the other side. Therefore the CME is pushed ahead by the initial magnetic pressure and the main part of the matter is pushed towards the shock front. Of course, for the unmagnetized CME there is no magnetic jail but also no magnetic pressure pushing the CME plasma ahead. In this case, the plasma blob evolves to a shape in between the two previous ones: not as "circular" as the inverse CME but not the flattened "pancake" of the normal CME either.

\subsection{The propagation velocity of the CMEs}

According to Zhang \& Golub (2003) a normal CME should be a fast event and an inverse CME should be a slow event. We can verify this by measuring the propagation velocity of the different CMEs. We distinguish two velocities: the velocity of the leading shock front and the velocity of the center of relative mass. The 1D center of relative mass on the equator is calculated as

$\operatorname{CORM}\left(\theta_{\mathrm{cme}}\right)=\left.\frac{\sum_{i} \bar{\rho}_{i} r_{i} \mathrm{~d} r_{i}}{\sum_{i} \bar{\rho}_{i} \mathrm{~d} r_{i}}\right|_{\theta_{\mathrm{cme}}}$

where $\bar{\rho}$ denotes the relative density $\left(\bar{\rho}=\left(\rho-\rho_{\text {wind }}\right) / \rho_{\text {wind }}\right)$ and the index $i$ run over all grid points in the radial directions. Figure 4 shows that in our simulations, the inverse CME propagates indeed slower than the normal CME. The velocity of the center of relative mass is $\pm 600 \mathrm{~km} \mathrm{~s}^{-1}$ for the inverse CME and $\pm 800 \mathrm{~km} \mathrm{~s}^{-1}$ for the normal CME. The center of relative mass of the initially unmagnetized CME propagates at a velocity in between these two. The velocities of the leading shock 

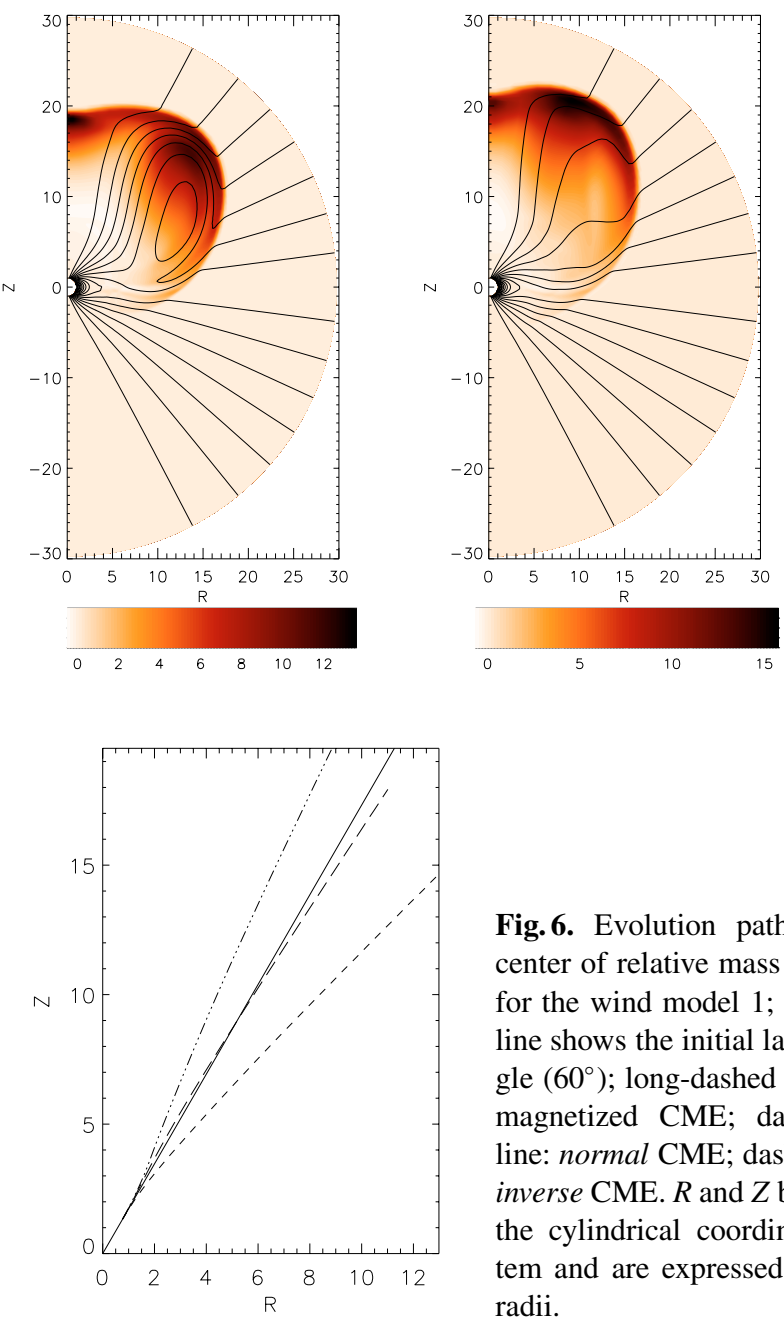

Fig. 6. Evolution path of the center of relative mass in space for the wind model 1 ; the solid line shows the initial launch angle $\left(60^{\circ}\right)$; long-dashed line: unmagnetized CME; dashed-dot line: normal CME; dashed line: inverse CME. $R$ and $Z$ belong to the cylindrical coordinate system and are expressed in solar radii.

fronts, however, are approximately the same for the three cases $\left( \pm 1000 \mathrm{~km} \mathrm{~s}^{-1}\right)$. The leading shock front of the normal CME propagates slightly faster than those of the inverse and the unmagnetized CME.

Also from the kinetic point of view, there are two different types of CMEs: the impulsive CME, with a huge acceleration near the sun and a constant velocity afterwards; and the gradual CME with a low but persistent acceleration (see Zhang et al. 2004). In our simulations, the normal CME looks like an impulsive $\mathrm{CME}$ and the inverse CME must be an intermediate event. This may be due to the lack of CME initiation model in our simulations. We just imposed the velocity (the same in all cases!) as an initial condition, without any acceleration mechanism.

\subsection{Analysis of the evolution path}

The evolution path followed by a CME is a very important characteristic of its propagation because it is a crucial parameter for space weather on Earth: a different evolution path may mean that the CME does not affect the Earth at all or affects it in a less direct or substantial way. When a CME is launched exactly on the equator, i.e. for $\theta_{\mathrm{cme}}=0^{\circ}$, no deviation is possible because of the symmetry. In order to study the effect of the

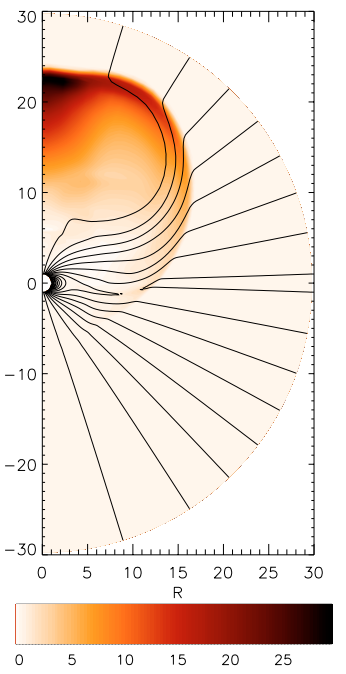

Fig. 5. Plots of the relative density distribution after $4 \mathrm{~h} 12 \mathrm{~min}$ for an initial launch angle of $60^{\circ}$; left: magnetized inverse; middle: unmagnetized; right: magnetized normal. The black lines represent the magnetic field.

magnetic field polarity on the evolution path of the CMEs, the CMEs are launched at $60^{\circ}$, out of the closed field lines region in the equatorial belt.

In order to study the evolution path, we define the $2 \mathrm{D}$ center of relative mass:

CORM $=\frac{\sum_{i j} \bar{\rho}_{i j} r_{i}^{2} \mathrm{~d} r_{i} \mathrm{~d} \theta_{j}}{\sum_{i j} \bar{\rho}_{i j} r_{i} \mathrm{~d} r_{i} \mathrm{~d} \theta_{j}}$

where the indices $i$ and $j$ run over all grid points in the radial and azimuthal directions, respectively. According to Figs. 5 and 6 , the initial polarity of the perturbation substantially affects the evolution path of the IP CMEs. For example, an inverse $\mathrm{CME}$ launched at $60^{\circ}$ swerves toward the equator while a normal CME veers toward the pole. The unmagnetized CME is not deviated and closely sticks to its initial launch angle. Hence, the deviation of the magnetized CMEs is due to the initial magnetic field configuration. In fact, as we have discussed before for the $\theta_{\text {cme }}=0^{\circ}$ case, the initial orientation of the magnetic pressure is very different for an inverse and for a normal CME. This is also true for the $\theta_{\mathrm{cme}}=60^{\circ}$ case. Figure $7 \mathrm{ex}-$ plains the reason of these different configurations, keeping in mind that because of Eq. (1) the extra magnetic field varies like $\frac{1}{R}$. It is obvious that for a normal CME, the initial magnetic pressure will push the perturbation away from the equator towards the pole. For an inverse CME, on the other hand, the initial magnetic pressure will push the perturbation towards the equator.

\subsection{The spread angle}

Another very important evolution parameter of a CME is its spread angle since, even if the center of the CME misses the earth, the wing of the CME can interact with the magnetosphere and create a severe geomagnetic storm. Therefore, we will try to quantify the influence of the initial polarity on the evolution of the spread angle of the corresponding CMEs. For this purpose, the CMEs are launched on the equator, because for a too large launch angle, an important part of the CME 

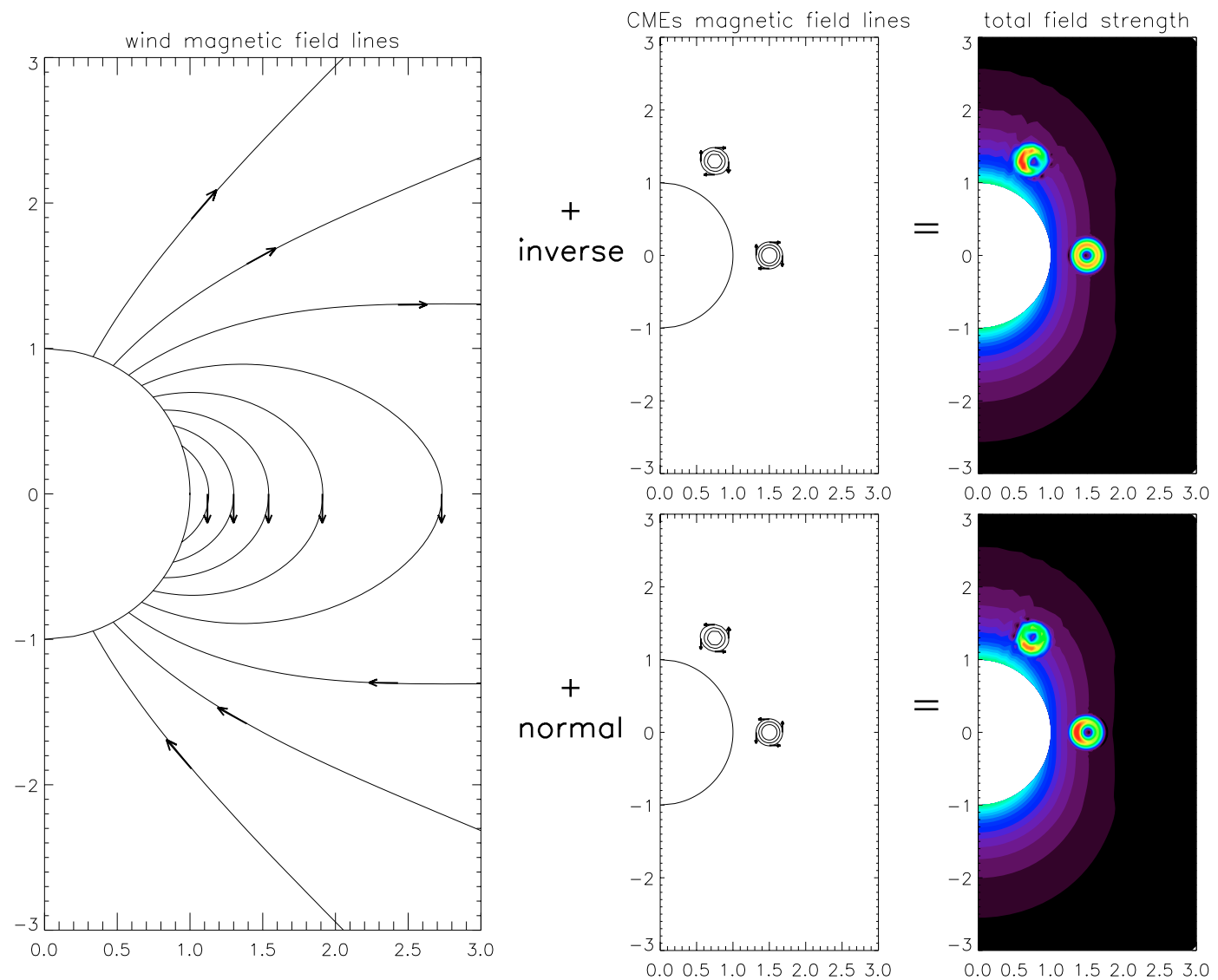

Fig. 7. Left: magnetic field lines of the background wind. Middle: magnetic field lines of the CMEs for the inverse (above) and the normal polarity (below). Right: the strength of the total magnetic field.

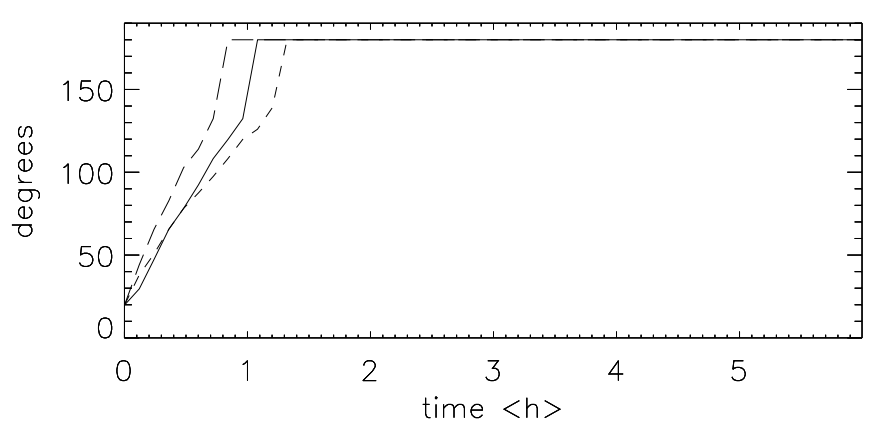

Fig. 8. The evolution of the spread angle of the CME in time, for a polytropic background wind (model 1). Long-dashed line: inverse CME; dashed line: normal CME; solid line: unmagnetized CME.

escapes from the computational domain, and it is impossible to define and measure a spread angle. We define the spread angle by means of the relative density plots on which we determine the contour where the relative density is one (i.e. $\rho=2 \rho_{\text {wind }}$ ). The spread angle of this contour measured from the solar center is defined as the spread angle of the CME. Remark that this definition is rather severe and much more strict than the usual way of determining the spread angle of real CMEs. But at least this definition has a mathematical basis and, therefore, can be reproduce.

Figure 8 shows the evolution of the spread angle in time. This evolution is not very different for the different CMEs in this case with a polytropic background wind: the spread angle quickly evolves to $180^{\circ}$. For the inverse CME this evolution is slightly faster than for an unmagnetized CME while for a normal CME it is slightly slower. However, below we will see that this effect is quite different for other background wind models. Generally, the inverse CMEs have a wider spread angle than the normal CMEs. The spread angle of the initially unmagnetized CME evolves in between these two extremes.

According to Fig. 3, for an inverse CME the bulk part of the plasma stays in the magnetic flux rope (small extension) but the small part which escapes from the flux rope (the shock front) spreads substantially. Probably a higher threshold for the relative density in our spread angle definition would yield a different picture.

\section{Effects of the background wind}

In this section we analyze similar simulations as above but in combination with different background solar wind models.

\subsection{Comparison of all CME-wind combinations}

In the present subsection we give and overview of all CME-wind combinations we simulated. We combined both the inverse and normal CME models with all three wind models described in the introduction and, in more detail, in Paper I. For comparison and for interpretation of the results, we also show 

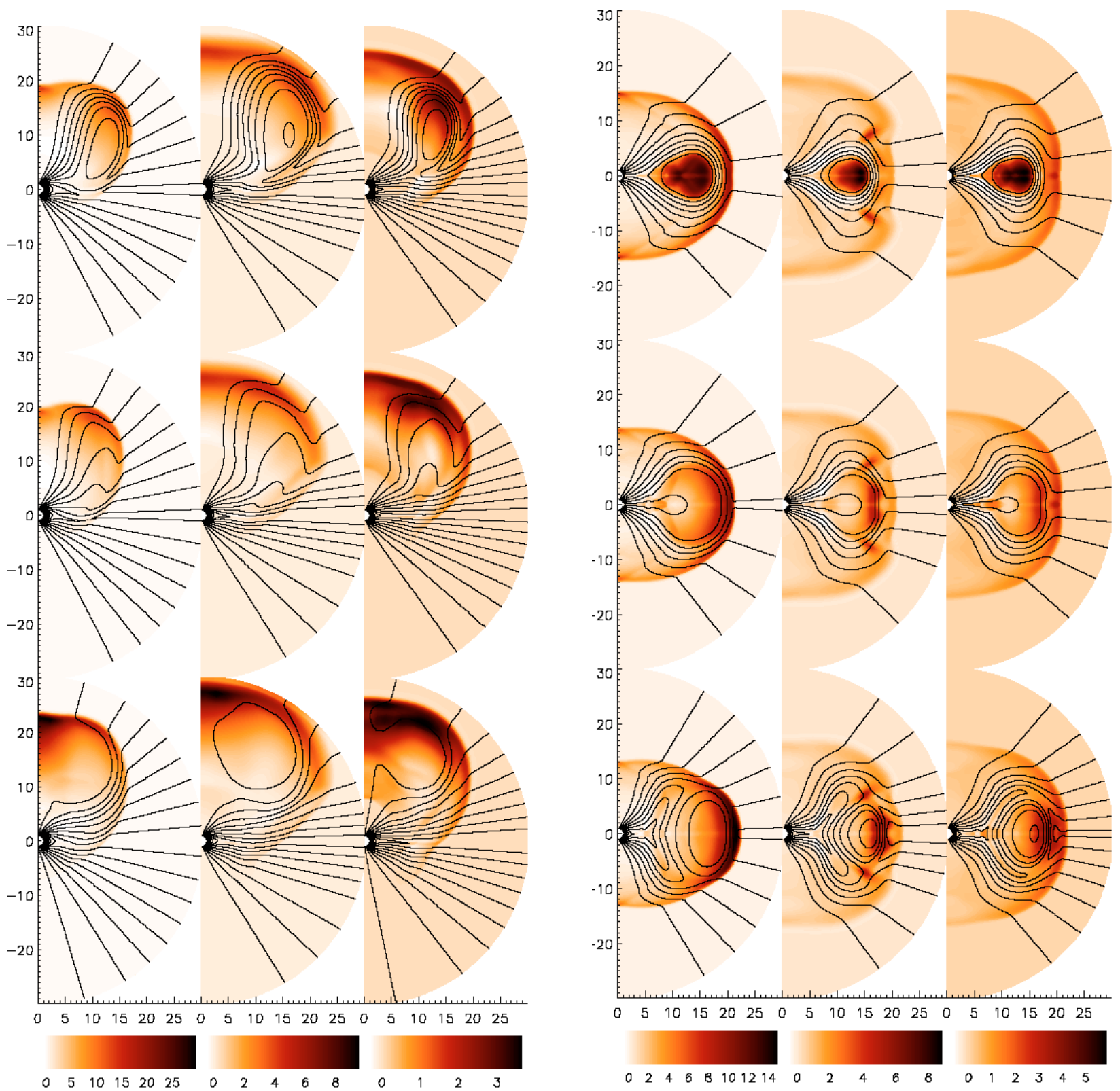

Fig. 9. Snapshots after $4 \mathrm{~h} 12 \mathrm{~min}$ of the relative density (color contour) and magnetic field lines (solid lines) for a CME with initial velocity $1000 \mathrm{~km} \mathrm{~s}^{-1}$ and $\theta_{\mathrm{cme}}=60^{\circ}$ (left panel) and $\theta_{\mathrm{cme}}=0^{\circ}$ (right panel) illustrating the influence of the background wind (horizontal) and the magnetic polarity of the CME flux rope (vertical). Lower rows: magnetized CME (normal polarity), Central rows: unmagnetized CME, Upper rows: magnetized CME (inverse polarity). Left columns: wind model 1, Central columns: wind model 2, Right columns: wind model 3.

the results for the unmagnetized CME again as a reference. We repeat that for all simulations we used exactly the same numerical technique, grid, boundary conditions, initial conditions, etc. Out of the extensive parameter study we made, below we discuss the results for two launch angles, $\theta_{\mathrm{cme}}=0^{\circ}$ and $60^{\circ}$, respectively. All CMEs have an initial velocity of $1000 \mathrm{~km} \mathrm{~s}^{-1}$.

Figure 9 gives an overview of the simulation results (snapshots after $4 \mathrm{~h} 12 \mathrm{~min}$ ) for CMEs launched on the equator and at $60^{\circ}$. Clearly, the background wind model used in the simulations has a substantial influence on the evolution of the CME. Nevertheless, all our conclusions about the importance of the initial polarity seem to hold for the other wind models as well, at least qualitatively. For each background wind model, the magnetized CMEs evolve to a magnetic field configuration that is in agreement with the predictions of Low \& Zhang (2002). For the three winds, the inverse CME launched on the equator results in a flux rope detached from the shock front. For each wind, a normal CME launched at an angle of $60^{\circ}$ is deviated to the pole and an inverse $\mathrm{CME}$ is deviated to the equator.

Figure 9 shows that not only the CME model (the initial polarity) is important, but also that the wind model plays an important role (see Paper I for a detailed study of the effect of the wind model on the unmagnetized CME). We repeated the above analysis for wind models 2 and 3 and, although the results are qualitatively the same, quantitatively they can differ quite substantially. For example, in Fig. 10 we show the 


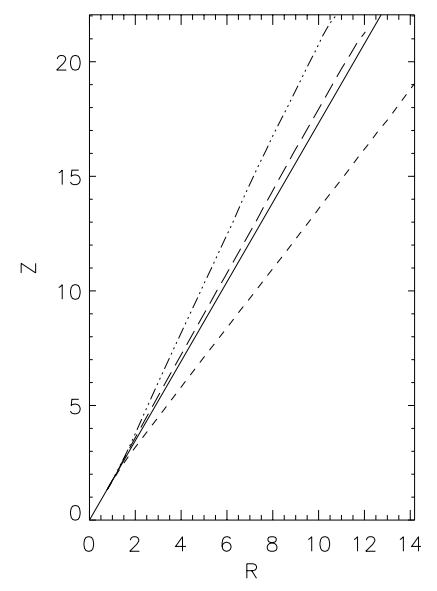

Fig. 10. Evolution path of the center of relative mass in space for the wind model 2; the solid line shows the initial launch angle $\left(60^{\circ}\right)$; long-dashed line: unmagnetized CME; dashed-dot line: normal CME; dashed line: inverse CME. $R$ and $Z$ belong to the cylindrical coordinate system and are expressed in solar radii.

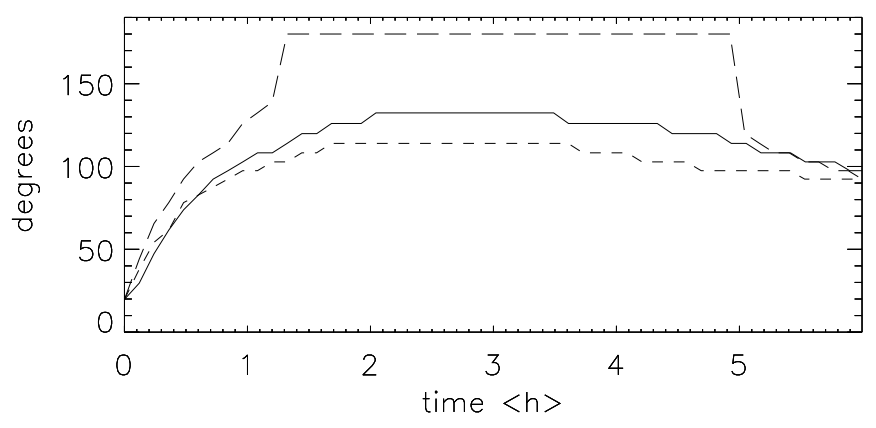

Fig. 11. The evolution of the spread angle of the CME in time, for the model 2 background wind. Long-dashed line: inverse CME; dashed line: normal CME; solid line: unmagnetized CME.

evolution path of the center of relative mass for the three CMEs launched at an angle of $60^{\circ}$ on top of wind model 2 . As for the wind model 1 (see Fig. 6) normal CME is deviated towards the pole (compared to the unmagnetized CME evolution path), while the inverse CME is deviated towards the equator, but not as much as in the wind model 1 . The unmagnetized $C M E$ again follows more or less its initial direction, although the path is now slightly above the $60^{\circ}$ coordinate line.

The background wind model substantially affects the spread angle of the CMEs. The effect is quantified for wind model 2 in Fig. 11, which is to be compared to Fig. 8 where we showed the results of the same analysis but for the model 1 background wind. We see that for wind model 2 the effect of the initial magnetic field of the CME is much more outspoken than for the model 1 wind. The spread angle grows again fastest for the inverse CME, but for the unmagnetized and the normal $\mathrm{CME}$ it now reaches a maximum between $100^{\circ}$ and $130^{\circ}$ after about two hours and then, after about $3.5 \mathrm{~h}$, it decreases again. Also for the inverse CME the spread angle decreases again after $5 \mathrm{~h}$, which was not the case for the polytropic wind (model 1).

\subsection{Interaction with the background wind}

In order to further quantify the differences between our $3 \mathrm{CME}$ models, a virtual satellite is placed in the equatorial plane at $15 R_{\odot}$ and the three different CMEs are launched on the equator, on top of the polytropic wind (model 1). This satellite registers $B_{Z}$ (the magnetic field component in the $Z$-direction), the density and the radial velocity as the CMEs pass by. The results are displayed in Fig. 12.

In this example, the signature of an inverse CME consists of an initial rapid decrease of $B_{Z}$ followed by an equally rapid increase, and of two peaks for the density profile corresponding with the passage of the leading shock front followed by the plasma blob captured in the flux rope. The normal CME, on the other hand, is characterized by an initial short increase of $B_{Z}$ and by a single sharp density peak (as the plasma blob is flattened to a "pancake" shape in this case). The unmagnetized CME has also a sharp density peak but for this CME model $B_{Z}$ has a negative peak, similar to the inverse CME case, but not as deep and not as wide. For all the three CME models, the wind is not in the original state at the end of the simulation $(36 \mathrm{~h})$. Hence, all three solar wind models take much longer to restore from the passage of a CME. As a result, when a second CME is emitted not too long after a first one, the first CME will influence the propagation of the second one. In this particulary case, the second CME will propagate on a higher speed wind and thus evolve differently than the first one.

Note that the data displayed in Fig. 12 mimic the measurements of a real satellite. Unfortunately, we cannot place this virtual satellite at $1 \mathrm{AU}$ because in our simulations the computational domain stops at $30 R_{\odot}$, as a result of our limited computer power.

\section{Conclusions}

We have tried to verify the effect of the initial magnetic field in the flux rope and of the background solar wind model on the evolution of the CME. For this purpose, we have superposed three different CME models (an unmagnetized, an inverse and a normal magnetized CME) on three different solar wind models: a polytropic wind, an MHD wind, and a polytropic wind with an Alfvén wave heating source term. The simulations are all performed in axi-symmetric geometry (2.5D) with the same numerical code, the same numerical technique and the same numerical grid (and thus the same numerical dissipation). We also used exactly the same boundary and initial conditions in this comparative study so that the study is objective and any difference in the evolution is entirely due to either the different initial magnetic field or the different background solar wind model used.

The CME simulations discussed in this paper demonstrate that the initial magnetic field polarity inside a flux rope plays a very important role in the evolution of CMEs. In particular, a different magnetic flux rope polarity leads to a different magnetic field configuration (the theoretical 'inverse' and 'normal' CME models of Low \& Zhang (2002) were confirmed), different shock speeds and different speeds of the CME plasma (quantified by the motion of the center of relative mass), a totally different mass distribution, significant differences in the spread angle of the CMEs, and, last but not least, considerably different evolution paths. To some extent, we were able to quantify these effects in the region $1-30 R_{\odot}$. 

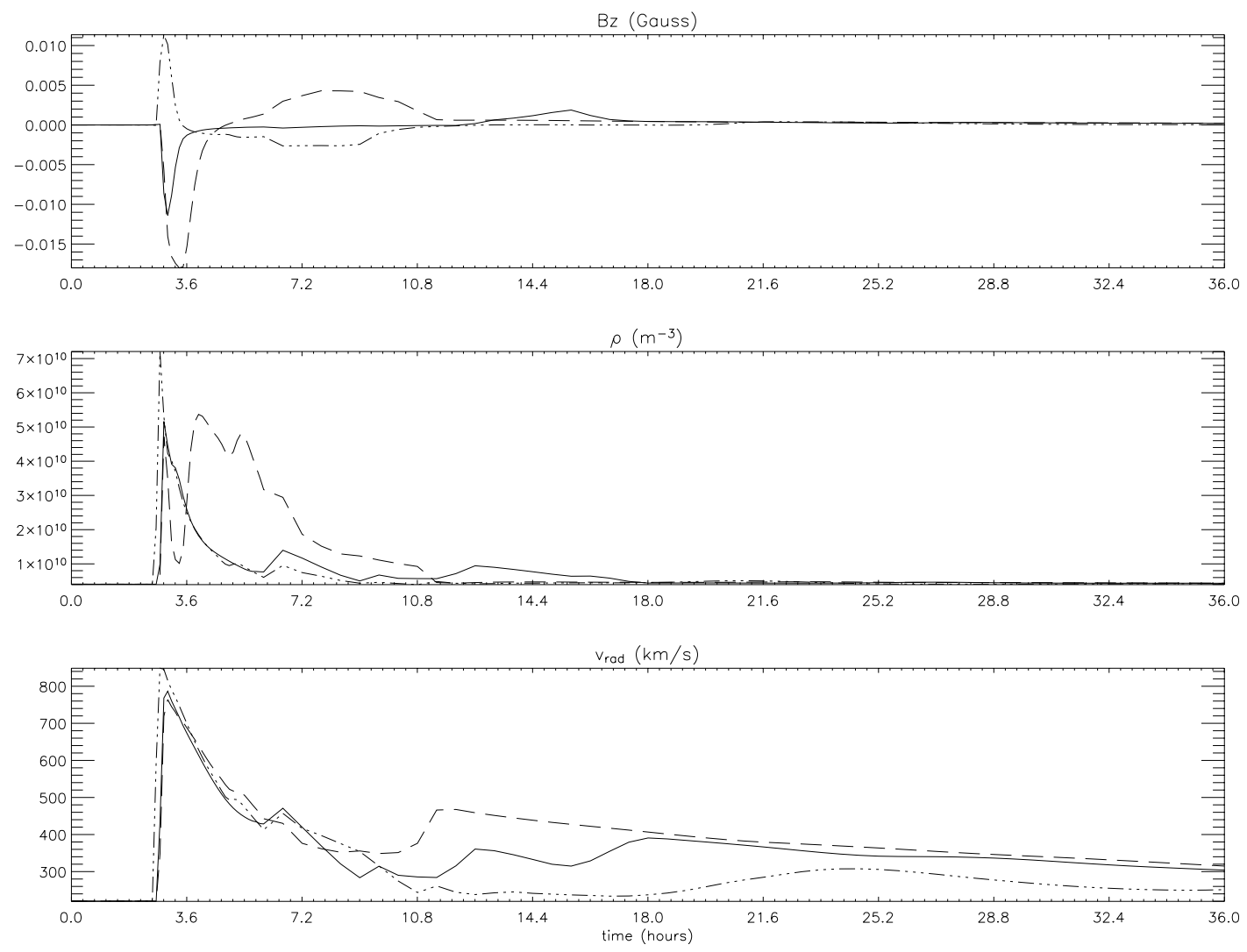

Fig. 12. Evolution of the magnetic field, density and velocity in time for a satellite in the equatorial plane at a position of $15 R_{\odot}\left(\theta_{\mathrm{cme}}=0^{\circ}\right)$; solid lines: unmagnetized CME; dash-dotted lines: normal CME; long dashes: inverse CME.

The present parameter study also revealed that the background wind model too substantially affects the evolution of the different CME models. Although the evolution of the different CME models is qualitatively the same for all three wind models, quantitatively the differences can be quite substantial. This means that both the initial conditions and the background wind need to be modelled with great care when space weather predictions are envisaged. The geo-effectiveness, and even whether the CME will hit the Earth or not, depends on the initial conditions and even a weak change in the initial magnetic field can make a lot of difference at $1 \mathrm{AU}$.

The latter remark brings us to next step of our parameter study. We urgently need to extend the computational domain to $1 \mathrm{AU}$ (or even more) so that we can measure the effect of the background wind and the magnetic field of the CME on the time of arrival at the Earth. Clearly, realistic simulations require the use of a 3D model both for the wind and for the CMEs. In fact, one should use real observational data to create a time dependent wind. In a next step, we would like to use more complex CME models, and include a CME initiation scenario.

Acknowledgements. These results were obtained in the framework of the projects OT/02/57 and GOA/2004/01 (K. U. Leuven), $14815 / 00 / \mathrm{NL} / \mathrm{SFe}$ (IC) (ESA Prodex 6), and the European Community's Human Potential Programme contract HPRN-CT2000-00153, PLATON, also acknowledged by B. vdH and E. C.

\section{References}

Gibson, S. E., \& Low, B. C. 1998, ApJ, 493, 460

Gombosi, T. I., DeZeeuw, D. L., Groth, C. P. T., Powell, K. G., \& Stout, Q. F. 2000, J. Atm. Terrest. Phys., 62, 1515

Groth, C. P. T., De Zeeuw, D. L., Gombosi, T. I., \& Powell, K. G. 2000, J. Geophys. Res., 25053

Jacobs, C., Poedts, S., van der Holst, B., \& Chané, E. 2004, in press

Keppens, R., \& Goedbloed, J. P. 1999, A\&A, 343, 251

Low, B. C., \& Zhang, M. 2002, ApJ, 564, L53

Manchester, W. B., Gombosi, T. I., Roussev, I., et al. 2004a, J. Geophys. Res. (Space Phys.), 109, 1102

Manchester, W. B., Gombosi, T. I., Roussev, I., et al. 2004b, J. Geophys. Res. (Space Phys.), 109, 2107

Odstrcil, D., Linker, J. A., Lionello, R., et al. 2002, J. Geophys. Res. (Space Phys.), 107, 14

Odstrcil, D., \& Pizzo, V. J. 1999, J. Geophys. Res., 104, 483

Odstrcil, D., \& Pizzo, V. J. 2002, in Proceedings of the Second Solar Cycle and Space Weather Euroconference, ESA SP-477: Solspa 2001, 293

Odstrcil, D., Riley, P., Linker, J. A., et al. 2003, in Solar Variability as an Input to the Earth's Environment, ESA SP-535, 541

Roussev, I. I., Forbes, T. G., Gombosi, T. I., et al. 2003, ApJ, 588, L45

Tóth, G. 1996, Astrophys. Lett. Commun., 34, 245

Wang, A. H., Wu, S. T., Suess, S. T., \& Poletto, G. 1995, Sol. Phys., 161,365

Zhang, J., Dere, K. P., Howard, R. A., \& Vourlidas, A. 2004, ApJ, 604, 420

Zhang, M., \& Golub, L. 2003, ApJ, 595, 1251 